\title{
Effect of Stearic Acid on the Rheology and Moulding Behaviour of 316 L Stainless Steel Powder Using Thermoplastic Natural Rubber (TPNR) Binder
}

\author{
Mohd Afian Omar ${ }^{1 *}$ and Norita Hassan ${ }^{2}$ \\ ${ }^{1}$ SIRIM Bhd., Lot 34, Jalan Hi Tech 2/3, Kulim Hi Tech Park, \\ 09000 Kulim, Kedah, Malaysia \\ ${ }^{2}$ Department of Manufacturing and Materials Engineering, \\ Faculty of Engineering, International Islamic University Malaysia, \\ 53100 Kuala Lumpur, Malaysia \\ *Corresponding author: afian@sirim.my
}

Published online: 31 December 2019

To cite this article: Mohd Afian Omar and Norita Hassan (2019). Effect of stearic acid on the rheology and moulding behaviour of $316 \mathrm{~L}$ stainless steel powder using thermoplastic natural rubber (TPNR) binder. Journal of Engineering Science, 15(2), 83-95, https://doi.org/10.21315/jes2019.15.2.6

To link to this article: https://doi.org/10.21315/jes2019.15.2.6

\begin{abstract}
In the present study, thermoplastic natural rubber (TPNR) has been developed as a novel backbone polymer to produce a new feedstock formulation for metal injection moulding (MIM) process. In this paper, the effect of stearic acid (SA) on the rheology properties and moulding behaviour has been investigated for the TPNR binder system. Feedstock mixture of $22 \mu \mathrm{m} 316 \mathrm{~L}$ stainless steel powder and the binder system consists of three major fractions of paraffin wax $(P W), T P N R$, and $S A$ with a powder loading of 65 vol. \% was investigated. Comparison also been made with existing of palm stearin (PS) in the binder system replacing the PW. The melt rheological behaviour of TPNR blends and the mixtures of the feedstock had been investigated. This study also investigates the effect of moulding temperature on the addition of SA in the binder formulation. The results show that the introduction of $10 \mathrm{vol}$. \% of SA in the binder that act as a lubricant helped in the mixing process to produce a homogeneous blend and the torque reached a steady state value in a short time. The viscosity of the feedstock also has been reduced with the lower injection moulding temperature.
\end{abstract}

Keywords: metal injection moulding, TPNR, paraffin wax, palm stearin, stearic acid 


\section{INTRODUCTION}

In the late 19th century, metal injection moulding (MIM) was then developed in order to cater for the production of small and complex shaped metal parts with outstanding mechanical properties. This technology offers several advantages over other precision fabrication process. The major advantage is that MIM can produce part with design flexibility of plastic injection moulding and lowcost alternative to the machining. In addition, MIM overcomes the productivity and dimensional limit of isostatic pressing and slip casting, limitations of the defects and tolerance in investment casting, the mechanical strength of die casting parts and the shape limitations of traditional powder compacts. ${ }^{1,2}$ Invariably, the MIM process consists of four steps that includes feedstock preparation by mixing of either metal powder with a binder, injection moulding to form a green part with the desired shape, debinding to form a brown part by removing the binder components, and sintering to near full density. Binder is the foundation in MIM component that supplies the metal powder flowability and formability necessary for moulding. Good binder should possess good flow characteristics, favourable interactions with the metal powder and good debinding characteristics as well as being low cost and environmentally friendly. ${ }^{1-4}$

In general, the thermoplastic polymers systems are the most studied and are used as the backbone polymer in industry. The high-density polyethylenewax binder is one of the simplest systems used in MIM. The main advantages are low cost, low molecular weight and good lubricating properties. Usually, multicomponent binders, comprising of polymer and other additives, are prepared because they improve the debinding process. The main advantage of these binders is the gradual elimination of different components preventing cracks and shape loss. The combination of low molecular weight and different polymer components allows a progressive removal as the temperature slowly increases. The combination of low density polyethylene (LDPE) and high density polyethylene (HDPE) in the LDPE/HDPE backbone polymer, can eliminate the article defect formed by the evaporation gas, and prevent the mass degradation of the backbone polymer in the articles, which frequently occurs when a single backbone polymer is present. ${ }^{1,2}$ Inspired by that, a combination of natural rubber (NR) and LDPE been produced known as thermoplastic natural rubber (TPNR) backbone polymer. TPNR was applied with paraffin wax (PW) and palm stearin (PS) forming a new binder system which is low viscosities and easily dissolved in organic solvent. The results on this investigation has been published elsewhere., 5 
In this article, the effect of stearic acid (SA) on the rheology properties and moulding behaviour has been investigated for the TPNR binder system. It is well known in the MIM process that SA can serve as a plasticiser for polymers, as a lubricant between powder and machine die walls and as a surfactant between powder and binder and claimed that a bulk mixture of powder and binder is difficult to obtain without the addition of SA in the binder system. Strongly preferred, adhesion of SA to the powder surface significantly reduces the binder/powder interfacial energy and contact angle but their usefulness increases as the particle surface area increases.

\section{EXPERIMENTAL}

\subsection{Feedstock Preparation}

To prepare the feedstock, initially the $316 \mathrm{~L}$ stainless steel powder was mixed with different formulation of binders while the volume fraction of the powder in the mixture was kept constant at $65 \%$. The variations compositions in weight fraction (wt. \%) of the PW and PS as a main binder system is shown in Table 1. Due to the homogeneity and flowability of feedstock containing 65 vol. \%, which gives near to full density sintered specimen, this powder volume fraction was chosen for further investigation. In order to investigate the role of SA in the binder, the effect of $10 \mathrm{wt}$ \% SA concentration on the feedstock properties was studied.

Table 1: Binder composition used to study the effect of varying the SA additions.

\begin{tabular}{lcccc}
\hline Label & SA (wt. \%) & PW (wt. \%) & PS (wt. \%) & TPNR (wt. \%) \\
\hline PW/TPNR & 0 & 55 & nil & 45 \\
PS/TPNR & 0 & nil & 55 & 45 \\
PW/TPNR/SA-1 & 10 & 55 & nil & 35 \\
PS/TPNR/SA-1 & 10 & nil & 55 & 35 \\
\hline
\end{tabular}

\subsection{Rheological Analysis}

Rheological properties of the feedstock was measured in a CFT-500D Shimadzu (Kyoto, Japan) capillary rheometer, using the standard procedure with temperature control of $\pm 0.5^{\circ} \mathrm{C}^{7}$ The piston velocity of the rheometer was varied (preheat for $120 \mathrm{sec}$ under 2, 3, 4, and $5 \mathrm{MPa}$ test load) and allowed to reach thermal equilibrium after charging the barrel. The experiment also occurred at different temperature testing. A tungsten carbide die with an orifice $1.0 \mathrm{~mm}$ in diameter $(D)$ and $10 \mathrm{~mm}$ in length $(L)$, giving a ratio $(L / D)$ of 10 was used (ASTM 
Standard D 3835-02). The softening temperature $\left(T_{s}\right)$ and beginning temperature flowability $\left(T_{b}\right)$ was investigated as a reference of the minimum temperature used for the characterisation. The experiment was conducted at the constant heating rate of $5^{\circ} \mathrm{C} \mathrm{min}{ }^{-1}$ from $80^{\circ} \mathrm{C}$ to $200^{\circ} \mathrm{C}$.

\subsection{Moulding Procedure}

The granulated feedstock of various formulations was injected into tensile bars using a simple, vertically aligned and pneumatically operated plunger machine, MCP HEK-GMBH (Lubeck, Germany). A barrel equipped with a thermocouple near the nozzle was heated by a jacket heater. Plunger type is considered an economical injection moulding machine as it saves materials used and also energy usage and is a simple machine. Feedstock was fed into the barrel and then injected through the nozzle in the mould cavity. In moulding experiments, the temperature and injection pressure were adjusted and the optimum conditions for each binder system was obtained. The green specimen was measured for green density and green strength. Morphology of the fracture moulded or green specimen has also been observed using scanning electron microscopy.

\section{RESULTS AND DISCUSSION}

\subsection{Rheological Behaviour}

It has been observed that the addition of $10 \%$ of SA in the binder formulations of PW/TPNR/SA-1 and PS/TPNR/SA-1 resulted a very small difference value of flow behaviour from the counterpart as shown in Figure 1. In contrast, feedstock without the existence of SA flow with lower viscosity value at the same temperature and pressure tested. These may be due to the higher composition of PW, PS, and TPNR in the binder system was good enough to generate the feasible feedstock. Even though, the presence of SA was expected as it functions as a lubricant in the mixing process to produce a homogeneous blend. This is because the SA helps the metal powder and the binder to have a better cohesion by increasing the wetting of the binder on the powder. Thus, it improves the injection mouldability of the feedstock.

The addition of a surfactant in the feedstock is said to help decrease the viscosity. By adding SA in the binder formulation, the viscosity and shear rate of feedstock will be decreased, and this would change the dilatant to pseudoplastic flow under applied stress at lower temperature. SA, as it forms a layer on the powder particle helps the powder to slide and then greatly reduces the viscosity. 
SA acts as a lubricant in the mixing process to produce a homogeneous blend. This indicates that addition of SA in the binder formulation plays a leading part in decreasing the viscosity where it reinforced the wetting property for the powder.

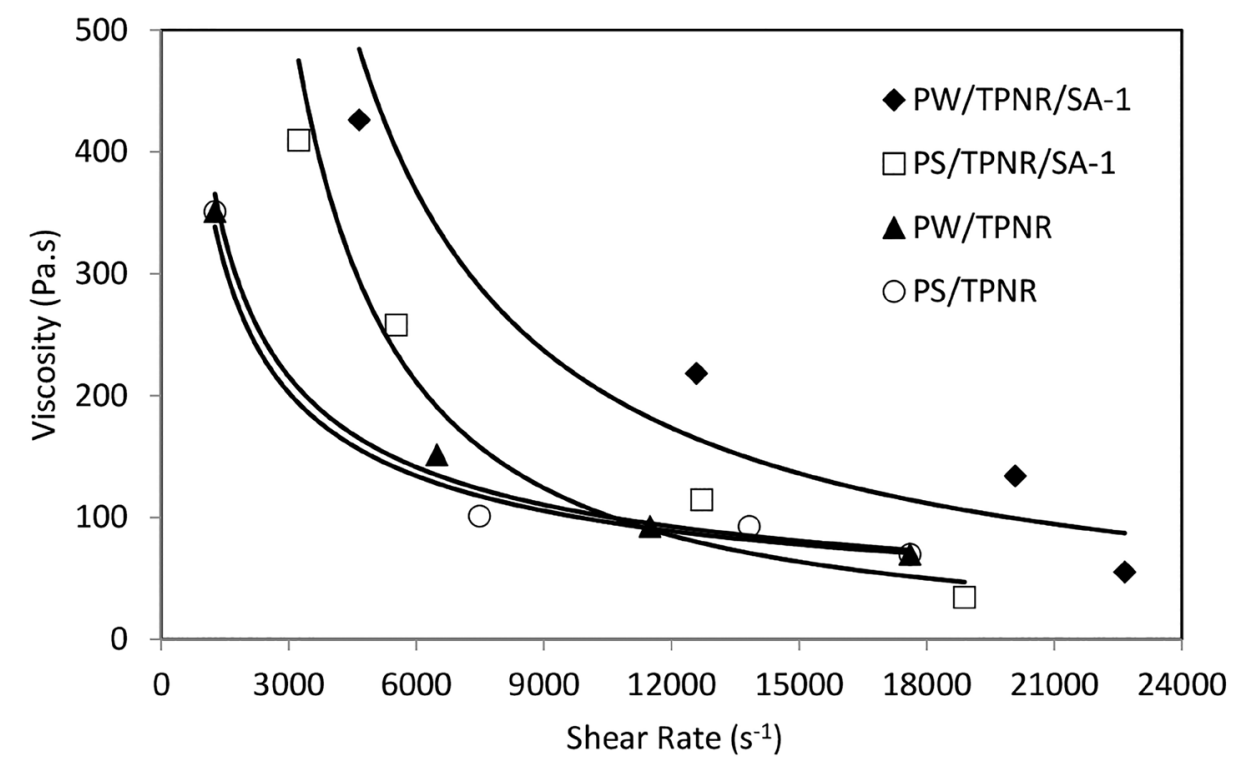

Figure 1: Effect of SA on the viscosity of feedstocks at $160^{\circ} \mathrm{C}$.

\subsection{Flow Activation Energy $(E)\left(\mathrm{kJ} \mathrm{mol}^{-1}\right)$}

Effect of temperature shifting is an important factor in MIM process. It should not be neglected as it plays important role to flow the feedstock into mould. By applying a suitable temperature, it will determine the successfulness of the injection moulding process. Moreover, the degradation of binder is also influenced by the temperature. An effect of temperature on viscosity is expressed by an Arrhenius equation, $\eta=\eta_{o} \exp (E / R T)$. The important parameter in this equation is the value of constant $E$ which refers to temperature dependency of viscosity. The higher value of $E$ indicates that the viscosity is too sensitive to temperature or otherwise. The value of $E$ should be as small as possible to avoid sharp viscosity changes that reduce the flowability of the feedstock and cause stress concentrations, cracking and distortion in the moulded parts.

Figure 2 shows the Arrhenius plot of viscosity against temperature at $50 \mathrm{~N}$ pressure applied. The data of the flow activation energy in Table 2 indicate that the feedstock contains of SA, i.e., feedstock PW/TPNR/SA-1 and PS/TPNR/ SA-1 giving higher flow activation energy rather than the feedstock system without 
SA, i.e. feedstock PW/TPNR and PS/TPNR. However, injection process of the feedstocks with presence of SA give smoother injection process as SA functions as a lubricant.

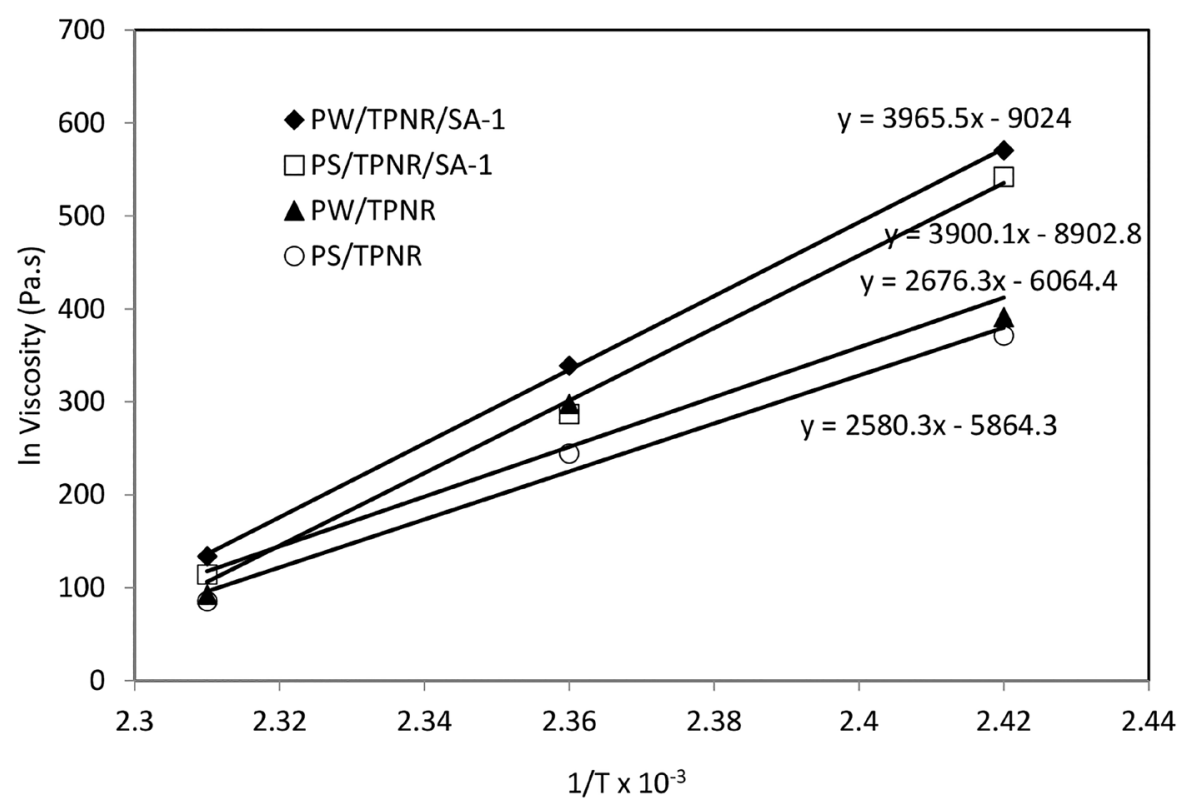

Figure 2: Correlation of viscosity and temperature for feedstock with different formulations at $50 \mathrm{~N}$ pressure.

The data obtained also point that the feedstock with binder system consist of PW or PS as one of the binder materials together with TPNR as the backbone polymer do not show much difference according to $E$ values as shown in Table 2 . The activation energy of feedstock PW/TPNR/SA-1 consists of 55 vol. \% PW and PS/TPNR/SA-1 consists of 55 vol. \% PS is 32.97 and $32.43 \mathrm{~kJ} \mathrm{~mol}^{-1}$, respectively. These values considered to be appropriate values, which imply that is the best from the standpoint of temperature sensitivity.

The value of $E$ indicates the influence of temperature on the viscosity of MIM feedstock. If the value of $E$ is low, the viscosity is not too sensitive to temperature differences, thus, any small fluctuation of temperature during injection moulding practice might not result on sudden viscosity change that can cause moulding defect. However, all formulations still considerably low, so the viscosity of feedstock composed of PW or PS with TPNR as the backbone polymer, with or without SA were giving impressive results and it is considered successfully developed as a new based binder system. 
Table 2: $E$ value for feedstock with different formulations of binder system at $50 \mathrm{~N}$ pressure.

\begin{tabular}{|c|c|c|c|c|}
\hline Formulation & $\begin{array}{l}\text { Temperature, } \\
(1 / T)\left(K^{-1}\right)\left(10^{-3}\right)\end{array}$ & $\begin{array}{l}\text { Viscosity } \\
\text { (Pa.s) }\end{array}$ & $\begin{array}{c}\text { Gradient } \\
(E / R)\end{array}$ & $\begin{array}{c}E \\
\left(\mathrm{~kJ} \mathrm{~mol}^{-1}\right)\end{array}$ \\
\hline \multirow[t]{3}{*}{ PW/TPNR/SA-1 } & 2.42 & 570.50 & 3,965 & 32.97 \\
\hline & 2.36 & 338.90 & & \\
\hline & 2.31 & 133.90 & & \\
\hline \multirow[t]{3}{*}{ PS/TPNR/SA-1 } & 2.42 & 792.00 & 3,900 & 32.43 \\
\hline & 2.36 & 287.00 & & \\
\hline & 2.31 & 114.30 & & \\
\hline \multirow[t]{3}{*}{ PW/TPNR } & 2.42 & 391.20 & 2,676 & 22.25 \\
\hline & 2.36 & 297.80 & & \\
\hline & 2.31 & 92.610 & & \\
\hline \multirow[t]{3}{*}{ PS/TPNR } & 2.42 & 371.20 & 2,580 & 21.45 \\
\hline & 2.36 & 244.50 & & \\
\hline & 2.31 & 85.61 & & \\
\hline
\end{tabular}

\subsection{Injection Moulding Parameters}

According to the observation during injection moulding process by using the vertical injection moulding machine, the parameter setting is not much different between the feedstock compounded at different formulations. This might be due to the constant powder loading at all formulation chosen. However, parameter observation shows that injection moulding process for feedstock with PW/TPNR/ SA-1 binder formulation successfully injected moulded at nozzle temperature of $195^{\circ} \mathrm{C}$ with $13-15 \mathrm{sec}$ per cycle time, and for feedstock with PS/TPNR/SA-1 binder formulation injected moulded at nozzle temperature of $190^{\circ} \mathrm{C}$ with cycle time of $10-12 \mathrm{sec}$.

Meanwhile for the feedstock of PW/TPNR and PS/TPNR binder formulation had been successfully injection moulded at $200^{\circ} \mathrm{C}$ with cycle time of 18-20 sec per cycle. Even though they are lower viscosities of both feedstocks are comparable with the other two systems apparently because of the higher fractions of the low molecular weight binder of PW and PS in the system; however, during injection moulding process the temperature is a bit higher because of the higher fraction of the backbone polymer binder (TPNR), which melted at the higher temperature. This can be proven according to the properties of feedstock as summarised in Table 3. Although there was variation of feedstock formulation, 
with suitable setting parameter, nevertheless all green specimens were fairly good and free from normal defects such as short shot, flashes at the parting surface, and binder separation.

Table 3: Moulding temperature at variant binder formulations.

\begin{tabular}{lcccc}
\hline Sample & PW/TPNR/SA-1 & PS/TPNR/SA-1 & PW/TPNR & PS/TPNR \\
\hline $\begin{array}{l}\text { Moulding temperature } \\
\left({ }^{\circ} \mathrm{C}\right)\end{array}$ & $195 \pm 1$ & $190 \pm 2$ & $200 \pm 2$ & $200 \pm 2$ \\
\hline
\end{tabular}

With the addition of a surfactant in the feedstock, it is possible to reduce the injection moulding temperature. The PW/TPNR/SA-1 feedstock made with 65 vol. \% with the composition of 55/35/10 and the PS/TPNR/SA-1 feedstock with the same composition was successfully injection moulded at $195^{\circ} \mathrm{C}$ and $190^{\circ} \mathrm{C}$, respectively, with an injection pressure of $30 \mathrm{MPa}$. By comparing with the injection temperature of feedstock prepared without an addition of SA, during observation it reduced the injection temperature from $5^{\circ} \mathrm{C}$ to $10^{\circ} \mathrm{C}$. Moreover, an addition of SA could increase the ability of the mixtures to flow, as indicated by the reduction in the moulding temperature.

\subsection{Green Density Evaluation}

Table 4 summarises the density of green specimen with SA compared to green specimen without SA addition. It was also found that the green specimens tend to be very soft and flexible and the specimen could easily be removed from the mould. Although an addition of SA improved mouldability, it was found that the green density was reduced. The green density for feedstock PS/TPNR/SA-1 was lowered by $4 \%$ from $5.4 \mathrm{~g} \mathrm{~cm}^{-3}$ to $5.2 \mathrm{~g} \mathrm{~cm}^{-3}$ for the mouldings made with the addition of 10 vol. $\%$ SA and for feedstock PW/TPNR/SA-1 the green density was decreased about $8 \%$ from $5.5 \mathrm{~g} \mathrm{~cm}^{-3}$ to $5.1 \mathrm{~g} \mathrm{~cm}^{-3}$. The reduction in green density was due to the less of binder wetting and covering the powder particle in which contributed to the voids growth among the powder particle of feedstock made with the addition of SA as it is easily degrade at higher temperature.

Table 4: Density for green part at variant binder formulations.

\begin{tabular}{lcccc}
\hline Sample & PW/TPNR/SA-1 & PS/TPNR/SA-1 & PW/TPNR & PS/TPNR \\
\hline Average density $\left(\mathrm{g} / \mathrm{cm}^{3}\right)$ & $5.2 \pm 0.4$ & $5.1 \pm 0.5$ & $5.5 \pm 0.4$ & $5.4 \pm 0.5$ \\
\hline
\end{tabular}




\subsection{Moulded Shrinkage Evaluation}

Figure 3 shows the effect of variation in binder formulation on the percentage of moulded shrinkage for specimen with binder formulation of PW/ TPNR/SA-1,PS/TPNR/SA-1, PW/TPNR, and PS/TPNR. Figure 3 expresses percent of shrinkage over dimensions of green specimens at variation of formulation used with constant 65 vol. \% powder loading. The differences in binder formulation and materials used will produce several different percentages of shrinkage. Generally, when a smaller volume of binder was used, a smaller shrinkage occurred during solidification. The percentage of the shrinkage measured also indicated that the shrinkage observed for the injected specimens were not uniform and was not according to the melted feedstock flow direction. There were four main dimensions measured; however, the most effected was the thickness dimension.

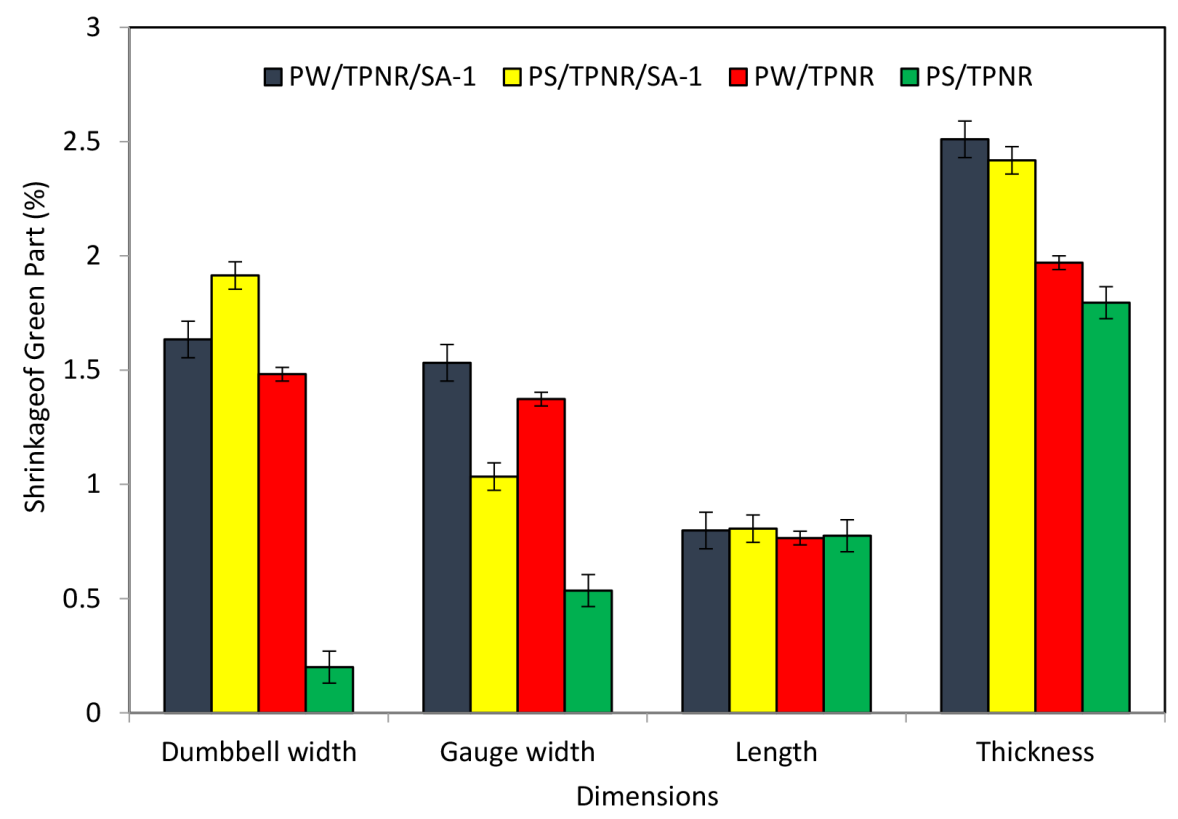

Figure 3: Shrinkage of green part at variant binder formulations.

Referring to previous study by Huang and Hsu (2009), they claimed that the feedstock based on LDPE performs worst in terms of the dimensional stability of green parts, because of the semi-crystalline LDPE materials, so exhibits a higher shrinkage rate $(0.7 \%-1.7 \%)$, which are comparable to the results measured for feedstock PW/TPNR/SA-1, PS/TPNR/SA-1, PW/TPNR, and PS/TPNR are in the range of $0.2 \%-2.5 \%$. Results obtained are significantly higher, which might be due to the higher composition of SA used. Moreover, shrinkage in green specimen 
occurs might be because of the existence of voids between the particles and due to entrapped air or binder shrinkage as the specimen cooled. Besides that, the activation energy value of each feedstock was also important as it influenced the cooling temperature in mould cavity.

Feedstock with higher value of the $E$ (PW/TPNR/SA-1 and PS/TPNR/SA1) will freeze faster as there is different apparent temperature among the nozzle and the mould cavity. Meanwhile, the feedstock with lower value of activation energy (PW/TPNR and PS/TPNR) needs longer time to freeze in mould cavity. This phenomenon can be related to flowability of the feedstock to fill the mould cavity. The value of $E$ should be as small as possible to avoid sharp viscosity changes that reduce the flowability of the feedstock and cause stress concentrations, cracking and distortion in the moulded parts. However, the percentage of the shrinkage was considered minimal as it can be accepted for further studies.

\subsection{Green Density}

Table 5 shows the effect of the different binder content on the green strength. From the data obtained it indicates that the green strength of specimen containing PW/TPNR were higher than the specimen consisting of PS/TPNR. Furthermore, the same trends also gained from both binder systems without the existence of SA (PW/TPNR and PS/TPNR). There is evidence from the green strength measurements that the PW content exhibited higher strength of the green specimens compared to PS content.

Table 5: Green strength of green part at different binder formulations.

\begin{tabular}{lcccc}
\hline Sample & PW/TPNR/SA-1 & PS/TPNR/SA-1 & PW/TPNR & PS/TPNR \\
\hline Green strength $(\mathrm{MPa})$ & $3.8 \pm 0.2$ & $1.6 \pm 0.2$ & $5.4 \pm 0.3$ & $2.5 \pm 0.1$ \\
\hline
\end{tabular}

The average green strength of PW/TPNR/SA-1 system is $3.8 \mathrm{MPa}$ and for $\mathrm{PW} / \mathrm{TPNR}$ system is about 5.4 MPa with $29.6 \%$ of differences in strength value. The green strength for PS/TPNR/SA-1 system is $1.6 \mathrm{MPa}$ and for PS/TPNR is $2.5 \mathrm{MPa}$ with $36 \%$ difference in the strength value. This phenomenon happened because of the existence of SA as surfactants, which act as lubricant. SA in the binder was preferentially adsorbed onto the surface of the powder particles and reduced the available sites to which the TPNR can bond to link the particles together, and as a result, the resistance to flow of the feedstock as well as the moulded strength was reduced. The evidence of SA adsorbed on the surface of the powder particles is shown in Figure 4 (white crystals). 

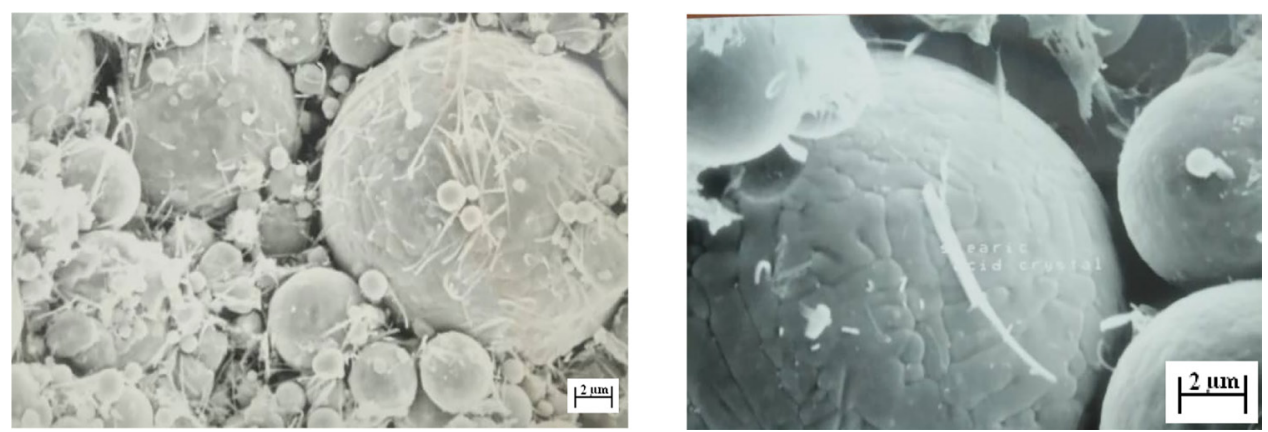

Figure 4: SEM micrograph shows the crystals of SA on the surface of the metal powder.

Figures 5(a) to (d) clearly shows the SEM of the green specimens at two different regions; at the fracture surface for four different binder systems. It can be seen that the binder fills practically all the interstitial spaces between the powder particles. The outer surface is filled with more binder than those of fracture surface. This is due to a greater contact of feedstock flow to the cavity wall and also that was the area to solidify first. Figure 5(a) clearly shows that the green specimen of PW/TPNR/SA-1 binder system is filled with more binder compared to PS/TPNR/ SA-1 binder system as shown in Figure 5(b). Even though the composition of the binder system is similar; it gives lower value in green strength. Many pores can be seen for the green specimens made from the binder system without the presence of SA. As it can be seen in Figures 5(a) and 5(b), especially at the fracture surface shows stiff fracture while for Figures 5(c) and 5(d) show ductile fracture, which indicates the higher strength of the green specimen. 


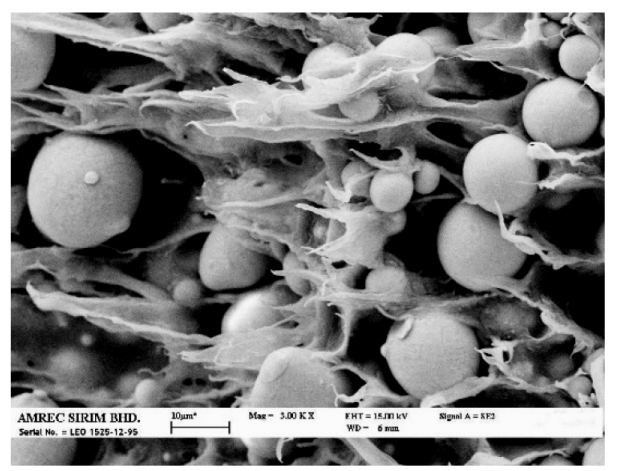

(a)

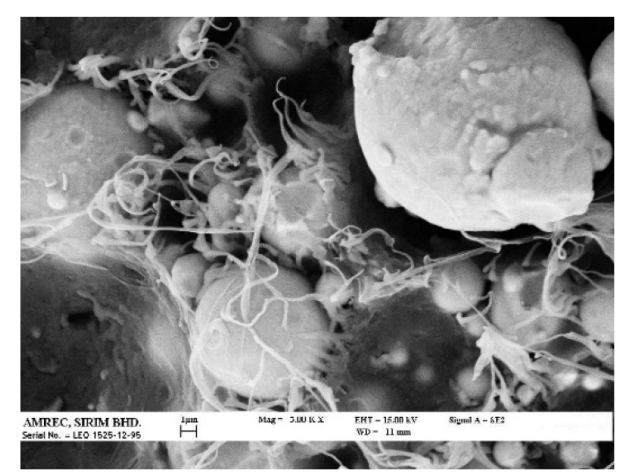

(c)

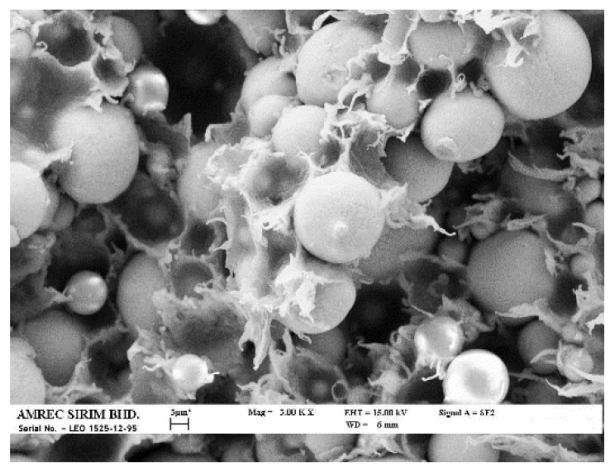

(b)

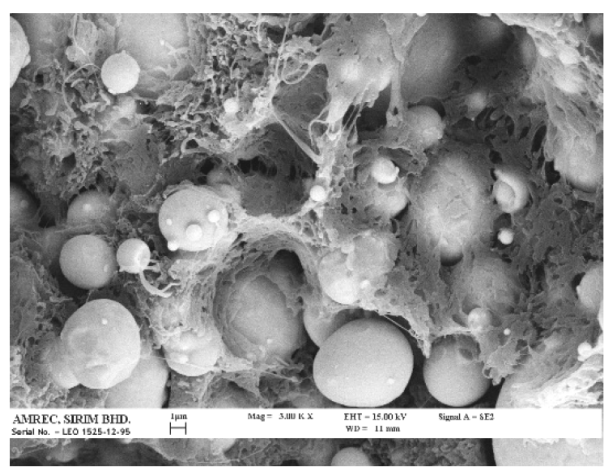

(d)

Figure 5: SEM micrograph of green parts fracture surface: (a) PW/TPNR/SA, (b) PS/TPNR/SA, (c) PW/TPNR, and (d) PS/TPNR.

\section{CONCLUSION}

The use of TPNR backbone polymer results in favourable flow behaviour of the MIM feedstock and thus improves the quality of the injection moulding. All formulations were successfully prepared and injection moulded without any defects. The addition of SA decreased the viscosity that resulted in reduction of the injection moulding temperature. However, in the binary binder system resulted lower viscosity as compared to the ternary system caused by the existence in higher volume percentage of low molecule binder (PW or PS) incorporation with higher volume percentage of TPNR backbone polymer. All feedstock showed their compatibility features according to the stability of the torque levels. SA added to the binder system as a lubricant reduces the viscosity and reduces the moulding temperature of the feedstock. SA in the binder was preferentially adsorbed onto 
the surface of the powder particles and reduced the available sites to which the backbone polymer can bond to link the particles together, and as a result, the resistance to flow of the feedstock and reduced the green strength of the specimens.

\section{ACKNOWLEDGEMENTS}

This study was supported by the Ministry of Energy, Science, Technology, Environment and Climate Change (MESTECC) Malaysia under the Technofund Grant.

\section{REFERENCES}

1. German, R. M. (1990). Powder injection molding. Princeton, NJ: Metal Powder Industries Federation (MPIF), 1-20.

2. German, R. M. \& Bose, A. (1997). Injection molding of metals and ceramics. Princeton, NJ: Metal Powder Industries Federation (MPIF), $1-30$.

3. Heaney, D. F., Mueller, T. J. \& Davies, P. A. (2004). Mechanical properties of metal injection moulded 316L stainless steel using both prealloy and master alloy techniques. Journal of Powder Metallurgy, 47(4), 367-373, https://doi.org/10.1179/003258904225020855.

4. Omar, M. A., Subuki, I., Abdullah, N. S., Mohd Zainon, N. \& Roslani, N. (2012). Processing of water-atomised 316L stainless steel powder using metal-injection processes. J. Eng. Sci., 8, 1-13.

5. Omar, M. A. \& Hassan, N. (2016). Development of thermoplastic natural rubber (TPNR) as a new binder in metal injection moulding. J. Eng. Sci., $12,43-51$.

6. Hassan, N., Ahmad, S., Muhamad, N., Omar, M. A. \& Hassan, N. A. (2013). Thermoplastic natural rubber (TPNR) as a backbone polymer for metal injection molding. Sains Malaysiana, 42(12), 1787-1791.

7. ASTM International (2002). ASTM Standard D 3835-02, Standard Test Method for Determination of Properties of Polymeric Materials by Means of a Capillary Rheometer. PA, USA: ASTM International, 486-496.

8. Huang, M. -S. \& Hsu, H. -C. (2009). Effect of backbone polymer on properties of $316 \mathrm{~L}$ stainless steel MIM compact. J. Mater. Process. Technol., 209(15-16), 5527-5535, https://doi.org/10.1016/j. jmatprotec.2009.05.011. 